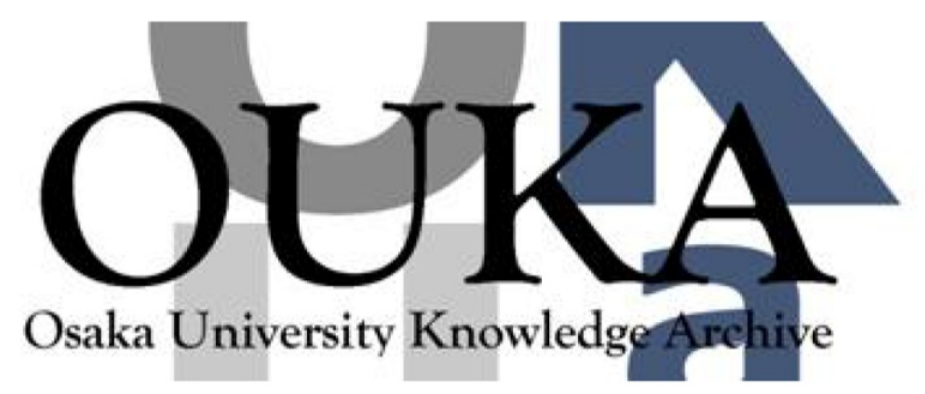

\begin{tabular}{|c|l|}
\hline Title & $\begin{array}{l}\text { Ponderomotive force and ion energy } \\
\text { distributions in an } r \text { sheath }\end{array}$ \\
\hline Author(s) & Hamaguchi, S.; Farouki, R. T.; Dalvie, M. \\
\hline Citation & Physical Review Letters. 68(1) p. 44-p. 47 \\
\hline Issue Date & $1992-01-06$ \\
\hline oaire:version & VoR \\
\hline URL & https://hdl. handle. net/11094/78516 \\
\hline rights & $\begin{array}{l}\text { Copyright (1992) by the American Physical } \\
\text { Society. }\end{array}$ \\
\hline Note & \\
\hline
\end{tabular}

Osaka University Knowledge Archive : OUKA

https://ir. Library. osaka-u. ac. jp/

Osaka University 


\title{
Ponderomotive Force and Ion Energy Distributions in an rf Sheath
}

\author{
S. Hamaguchi, R. T. Farouki, and M. Dalvie \\ IBM Thomas J. Watson Research Center, Yorktown Heights, New York 10598
}

(Received 26 August 1991)

\begin{abstract}
The incident-ion energy distribution in a collisionless $\mathrm{rf}$ sheath is derived analytically for a general electric-field configuration in the high-frequency regime. The analysis is based on a two-time-scale asymptotic expansion of the ion equation of motion, where the ratio of the ion transit frequency $\omega_{\mathrm{tr}}$ to the $\mathrm{rf}$ frequency $\omega$ is assumed to be small. It is shown that the "ponderomotive force" due to the rf modulation of the electric field exerts a retarding effect on the ion motion, counteracting the dc-bias field. The results obtained here are applicable to $\mathrm{rf}$-discharge-based process tools used in microelectronics fabrication, where the conditions of low collisionality and high rf frequency are usually satisfied.
\end{abstract}

PACS numbers: 52.20.Dq, 51.10.+y, 51.50.+v, 52.80.Vp

Radio-frequency (rf) discharges [1-5] play a central role in integrated-circuit fabrication processes, such as sputtering, etching, and chemical vapor deposition. The outcome of these processes is known to be a sensitive function of the ion bombardment energy on the electrodes, which is acquired by ions as they traverse the sheath field $[6,7]$. In various plasma environments ranging from diode glow discharges to electron cyclotron resonance (ECR) generated plasmas, it has been observed that the time-averaged energy distribution of ions impinging on the cathode surface has a double-peaked, "hollow" profile $[8,9]$.

Although various numerical calculations have reproduced such profiles for the ion energy distribution in $\mathrm{rf}$ sheaths [10-14], few analytical attempts have been made to derive the ion distribution corresponding to a general $\mathrm{rf}$ electric-field profile. For example, Benoit-Cattin and Bernard [15] derived the ion distribution in a special case, in which the amplitude of the rf field is sufficiently small compared to that of the dc-bias field, and the space dependence is given by the collisionless Child-Langmuir law [16] $\left[E \propto(1+\lambda \cos \omega t) z^{1 / 3}\right.$, with $\left.\lambda \ll 1\right]$.

In most rf-discharge process tools, however, the sheath electric field is more complex: The spatial dependence is not necessarily a simple function of $z$ and the amplitude of the rf field may be comparable to that of the dc-bias field. On the other hand, various experiments and numerical calculations have revealed that regimes of low collisionality $\left(d / \lambda_{\mathrm{MFP}} \ll 1\right.$, where $d$ denotes the sheath thickness and $\lambda_{\text {MFP }}$ the ion mean free path) and high frequency $\left(\tau_{\text {tr }} \omega \ll 1\right.$, where $\tau_{\text {tr }}$ denotes the ion-transit time) in an $\mathrm{rf}$ sheath lead to a well-controlled, narrow energy distribution of the bombarding ions. Therefore, in the present work, we focus on a collisionless, high-frequency $\mathrm{rf}$ sheath, with the rf electric field of a general functional form $E(z, \omega t)$.
Before discussing general electric-field profiles, we begin by considering ion motion in a sinusoidally oscillating electric field $E(z, \omega t)=E_{0}(z)+E_{1}(z) \cos \omega t$ with arbitrarily space-dependent $E_{0}(z)$ and $E_{1}(z)$. This may be regarded as the lowest-order approximation in the Fourier expansion to a general field.

Using the normalized variables $\tau=\omega\left(t-t_{l}\right), \zeta=z / d$, and $\epsilon^{2}=q E_{0}(d) / m d \omega^{2}$, the equation of motion for the ion at position $z$ is given by

$$
\frac{d^{2} \zeta}{d \tau^{2}}=\epsilon^{2}\left[F_{0}(\zeta)+F_{1}(\zeta) \cos (\tau+\phi)\right],
$$

where $\phi=\omega t_{l}, F_{0}=E_{0} / E_{0}(d)$, and $F_{1}=E_{1} / E_{0}(d)$. For simplicity, the ions are assumed to be injected into the sheath with the Bohm velocity $v_{l}=\left(k_{B} T_{e} / m\right)^{1 / 2}$ at position $z=0$ and at any given time $t=t_{l}$. The initial conditions then become $\zeta=0$ and $u=d \zeta / d \tau=u_{I} \quad\left(v_{z}=d z / d t\right.$ $\left.=v_{l}\right)$ at $\tau=0$. For each $\phi$, the corresponding ion trajectory is obtained by integrating Eq. (1) until $\zeta=1$, the location of the cathode. Here we may define a nominal "ion transit time" by $\tau_{\mathrm{tr}}=\left[m d / q E_{0}(d)\right]^{1 / 2}$, which typically gives a lower bound on the true transit time across the sheath, since $E_{0}(z)$ is generally a monotonically increasing function in a simple sheath. We also define an "ion transit frequency" by $\omega_{\mathrm{tr}}=\tau_{\mathrm{tr}}^{-1}$. It follows that $\epsilon=\omega_{\mathrm{tr}} / \omega$, which is small in a high-frequency rf system.

Consider first the solution to the above equation in the limit $\epsilon \rightarrow 0$. Let $\zeta_{0}(\tilde{\tau})$ with $\tilde{\tau}=\epsilon \tau$ satisfy

$$
\frac{d^{2} \zeta_{0}}{d \tilde{\tau}^{2}}=F_{0}\left(\zeta_{0}\right)
$$

so that $d^{2} \zeta_{0} / d \tau^{2}=\epsilon^{2} F_{0}\left(\zeta_{0}\right)$. Thus $\zeta_{0}$ represents the (normalized) ion trajectory in the limit of infinite frequency, where the ion feels only the dc component of the electric field. The correction of the trajectory due to finite $\epsilon$ is then obtained by writing $\zeta(\tau)=\zeta_{0}(\epsilon \tau)+\epsilon^{2} \xi(\tau)$, where, from Eq. (1), $\xi$ satisfies

$$
\frac{d^{2} \xi}{d \tau^{2}}=F_{1}\left(\zeta_{0}\right) \cos (\tau+\phi)+\epsilon^{2} \xi\left[F_{0}^{\prime}\left(\zeta_{0}\right)+F_{1}^{\prime}\left(\zeta_{0}\right) \cos (\tau+\phi)\right]+\mathcal{O}\left(\epsilon^{4}\right) .
$$

Here, primes denote derivatives with respect to $\zeta$, i.e., $F_{1}^{\prime}=d F_{1} / d \zeta$.

It should be noted that the right-hand side of Eq. (3) includes both a rapidly time-varying quantity, $\cos (\tau+\phi)$, and slowly time-varying terms-those that depend on $\zeta_{0}(\tilde{\tau})$. In order to solve Eq. (3), therefore, we employ a two-time- 
scale expansion [17] of the function $\xi$, i.e., $\xi=\xi_{0}(\tilde{\tau}, \tau)+\epsilon \xi_{1}(\tilde{\tau}, \tau)+\epsilon^{2} \xi_{2}(\tilde{\tau}, \tau)+\cdots$, where $\tilde{\tau}=\epsilon \tau$ denotes the slowvarying part of the time variable, while $\tau$ is the fast-varying part. Noting that $d / d \tau=\epsilon \partial / \partial \tau+\partial / \partial \tau$, we obtain from Eq. (3)

$$
\frac{d^{2} \xi}{d \tau^{2}}=\frac{\partial^{2} \xi_{0}}{\partial \tau^{2}}+\epsilon\left(2 \frac{\partial^{2} \xi_{0}}{\partial \tilde{\tau} \partial \tau}+\frac{\partial^{2} \xi_{1}}{\partial \tau^{2}}\right)+\epsilon^{2}\left(\frac{\partial^{2} \xi_{0}}{\partial \tilde{\tau}^{2}}+2 \frac{\partial^{2} \xi_{1}}{\partial \tilde{\tau} \partial \tau}+\frac{\partial^{2} \xi_{2}}{\partial \tau^{2}}\right)+\mathcal{O}\left(\epsilon^{3}\right)
$$

On equating the right-hand sides of Eqs. (3) and (4), we have, to the lowest order in $\epsilon$,

$$
\frac{\partial^{2} \xi_{0}}{\partial \tau^{2}}=F_{1}\left(\zeta_{0}\right) \cos (\tau+\phi),
$$

the solution of which is given by

$$
\xi_{0}=-F_{1}\left(\zeta_{0}\right) \cos (\tau+\phi)+A_{0}(\tilde{\tau}) \tau+B_{0}(\tilde{\tau}) .
$$

Since we assume that $\xi_{0}$ is of order unity, we require that the secular term (the second term of the right-hand side) of Eq. (5) vanish, i.e., $A_{0} \equiv 0$. The third term $B_{0}(\tilde{\tau})$ will be determined by a higher-order equation. In what follows, however, the explicit form of $B_{0}$ is not required, so the calculation of $B_{0}(\tilde{\tau})$ will be presented elsewhere.

We now calculate the time-averaged force exerted on an ion. Defining a time-averaging operation in the fast time scale by

$$
\bar{f}(\tilde{\tau})=\frac{1}{2 \pi} \int_{0}^{2 \pi} f(\tilde{\tau}, \tau) d \tau,
$$

and using Eq. (3), we calculate

$$
\begin{aligned}
\frac{\overline{d^{2} \zeta}}{d \tau^{2}}= & \epsilon^{2} \frac{d^{2} \zeta_{0}}{d \tilde{\tau}^{2}}+\epsilon^{2} \frac{\overline{d^{2} \xi}}{d \tau^{2}} \\
= & \epsilon^{2} F_{0}\left(\zeta_{0}\right) \\
& +\epsilon^{4}\left[B_{0} F_{0}^{\prime}\left(\zeta_{0}\right)-\frac{1}{2} F_{1}^{\prime}\left(\zeta_{0}\right) F_{1}\left(\zeta_{0}\right)\right]+\mathcal{O}\left(\epsilon^{6}\right),
\end{aligned}
$$

where we have used the expression for $\xi_{0}$ given by Eq. (5), and the fact that $\overline{\cos (\tau+\phi)}=0$ and $\cos ^{2}(\tau+\phi)=\frac{1}{2}$. Since the average position of the ion at time $\tilde{\tau}$ is given by $\bar{\zeta}=\zeta_{0}+\epsilon^{2} \bar{\xi}_{0}+\mathcal{O}\left(\epsilon^{3}\right)=\zeta_{0}+\epsilon^{2} B_{0}+\mathcal{O}\left(\epsilon^{3}\right)$, we may write $F_{0}(\bar{\zeta})=F_{0}\left(\zeta_{0}\right)+\epsilon^{2} B_{0} F_{0}^{\prime}\left(\zeta_{0}\right)$, up to order $\epsilon^{2}$. Using Eqs. (4) and (6), we also obtain $\overline{d^{2} \zeta / d \tau^{2}}=\epsilon^{2} d^{2} \bar{\zeta} / d \tilde{\tau}^{2}$ $+\mathcal{O}\left(\epsilon^{3}\right)$. Therefore, the equation for the average ion trajectory becomes

$$
\frac{d^{2} \bar{\zeta}}{d \tilde{\tau}^{2}}=F_{0}(\bar{\zeta})-\frac{\epsilon^{2}}{4} \frac{d F_{1}^{2}}{d \zeta}(\bar{\zeta})+\mathcal{O}\left(\epsilon^{3}\right)
$$

In terms of a unit volume with ion density $n_{i}$, Eq. (8) may be written, up to order $\epsilon^{2}$, as

$m n_{i} \frac{d^{2} \bar{z}}{d t^{2}}=q n_{i} E_{0}(\bar{z})-\left.\left(\frac{\omega_{p i}}{\omega}\right)^{2} \frac{d}{d z}\left(\frac{\epsilon_{0} E_{1}^{2}}{4}\right)\right|_{z=\bar{z}}$,

where $\omega_{p i}=q\left(n_{i} / m \epsilon_{0}\right)^{1 / 2}$ denotes the ion plasma frequency. The last term in Eq. (9) represents a force proportional to the time-averaged pressure (or the energy density) of the oscillating field $E_{1} \cos \omega t$, which is known as the ponderomotive force or Miller's force [18]. Since the sheath electric field is generally an increasing function of $z$, the sense of the ponderomotive force is opposite to that of the dc field $E_{0}$; i.e., it retards the ion motion through the sheath from the plasma bulk to the cathode.

From Eq. (5), the velocity $u$ at time $\tau$ may be calculated as

$u(\tau)=\frac{d \zeta}{d \tau}=\epsilon u_{0}(\epsilon \tau)+\epsilon^{2} F_{1}\left(\zeta_{0}\right) \sin (\tau+\phi)+\mathcal{O}\left(\epsilon^{3}\right)$,

where $u_{0}(\tilde{\tau})=d \zeta_{0}(\tilde{\tau}) / d \tilde{\tau}$. Denoting the time-averaged, normalized potential by $V_{0}(\zeta)$, i.e., $F_{0}=-d V_{0} / d \zeta$, we have from Eq. (2)

$$
\epsilon u_{0}=\left[u_{I}^{2}-2 \epsilon^{2} V_{0}\left(\zeta_{0}\right)\right]^{1 / 2} \text {. }
$$

By substituting $\zeta_{0}=\zeta-\epsilon^{2} \xi_{0}$ into Eqs. (10) and (11) and taking the terms up to order $\epsilon^{2}$, we obtain the final velocity if of the ion (i.e., its velocity when it reaches the cathode at $z=d$ ) in dimensional form as

$v_{f}=\left[v_{l}^{2}-2 q \psi_{0}(d) / m\right]^{1 / 2}+\left[q E_{1}(d) / m \omega\right] \sin \omega t$.

The ion (velocity) distribution function $f\left(t, z, v_{z}\right)$ at position $z$ in a collisionless sheath satisfies the Vlasov equation [18] with the boundary condition $f\left(t, z=0, v_{z}\right)$ $=n_{i} \delta\left(v_{z}-v_{l}\right)$. The characteristics of the Vlasov equation are determined by the equation of motion, i.e., Eq. (1), and the standard method of characteristics leads to the solution at $z=d$ given by

$$
f\left(t, d, v_{z}\right)=\left(n_{i} v_{l} / v_{0 f}\right) \delta\left(v_{z}-v_{f}\right),
$$

where $v_{0 f}=\left[v_{l}^{2}-2 q \psi_{0}(d) / m\right]^{1 / 2}$ denotes the zeroth-order velocity $v_{0}\left(=d \omega u_{0}\right)$ at the cathode $z=d$. The energy distribution $\Gamma_{\epsilon}$ of the ion flux at the cathode is given by $\Gamma_{\mathscr{E}} d \mathscr{E}=v_{z} f\left(t, d, v_{z}\right) d v_{z}$, namely, $\Gamma_{\mathscr{E}}=\left(n_{i} v_{l} / m v_{0 f}\right)$ $\times \delta\left(\sqrt{2 \mathscr{E} / m}-v_{f}\right)$ with the kinetic energy $\mathscr{E}=m v_{z}^{2} / 2$. The experimentally observed ion energy flux distribution is the time average of $\Gamma_{\mathscr{G}}$ :

$$
\bar{\Gamma}=\frac{\omega}{2 \pi} \int_{0}^{2 \pi / \omega} \Gamma_{G} d t=\frac{n_{i} v_{I}}{2 \pi v_{0 f}} \int \frac{\delta(g) d g}{d g / d \varphi},
$$

where $\varphi=\omega t$ and $g=\sqrt{2 \mathscr{E} / m}-v_{f}$. Note that the condition $g=0$ generally identifies two values of $\varphi$ in $0 \leq \varphi \leq 2 \pi$. Carrying out the integration in Eq. (14), we obtain

$$
\bar{\Gamma}(\mathscr{E})=\frac{n_{i} v_{I}}{\pi m v_{0 f}\left[\left(v_{+}-\sqrt{2 \mathscr{E} / m}\right)\left(\sqrt{2 \mathscr{E} / m}-v_{-}\right)\right]^{1 / 2}},
$$

where $v^{\prime} \pm=v_{0 f} \pm q E_{1}(d) / m \omega$.

The energy distribution $\bar{\Gamma}$ given in Eq. (15) has singu- 
larities at $\mathscr{E}=\mathscr{E}_{+}$and $\mathscr{E}_{-}$, where $\mathscr{E}_{ \pm}=m\left[v_{0} \pm q E_{1}(d) /\right.$ $m \omega]^{2} / 2$ and these singular points also define the limits of the energy spread. The width of the energy spread $\Delta \mathscr{E}=\mathscr{E}_{+}-\mathscr{E}_{-}$is thus given by $\Delta \mathscr{E}=\left[2 q E_{l}(d) / \omega\right]\left[v_{l}^{2}\right.$ $\left.-2 q \psi_{C}(d) / m\right]^{1 / 2}$ with $v l_{l}^{2}=k_{B} T_{e} / m$. In particular, when $k_{B} T_{e} \ll-q \psi_{C}(d)$, then

$$
\Delta \mathscr{E}=\left[2 q E_{1}(d) / \omega\right](2 q V / m)^{1 / 2},
$$

where $V=-\psi_{C}(d)$ denotes the dc-bias sheath potential.

The analytical expression, Eq. (15), has been compared to numerical simulations with various electric-field profiles and found to be in good agreement. For example, consider the case $E_{0}(z)=E_{1}(z)=E_{C}(z)$, where $E_{C}(z)$ $=-d \psi_{C} / d z$ is the collisionless sheath field. Denoting the ion density and the electric field at the presheath-sheath boundary by $n_{I}$ and $E_{I}$, the potential $\psi_{C}(z)$ is known to satisfy

$$
\frac{d^{2} \chi}{d \zeta^{2}}=-\frac{\alpha^{2}}{\sqrt{1-\chi}} \text { with } \chi(0)=0, \frac{d \chi}{d \zeta}(0)=-\beta,
$$

where $\chi=2 q \psi_{C} / m v_{l}^{2}, \quad \alpha=\sqrt{2} d / \lambda_{D}, \quad \lambda_{D}=\epsilon_{0} m / q^{2} n_{l}$, and $\beta=2 d q E_{l} / m v^{\prime}$. The closed-form expression for $E_{C}(z)$, which is rather complicated, is given in [19]. The timeperiodic electric field $E(z, \omega t)=E_{C}(z)(1+\cos \omega t)$ is illustrated in Fig. 1. With the initial velocity $u_{I}=v_{l} / d \omega$ $=0.02$ and the small parameter $\epsilon=0.16$, the analytical expression for the energy distribution, Eq. (15), is shown in Fig. 2(a), where $\mathscr{E}_{\max }=-2 q \psi_{C}(d)$. Figure 2(b) shows the direct integration of Eq. (1) with uniformly distributed random initial phase $\phi=\omega t_{I}$ for the same parameters, obtained from a simulation program similar to the Monte Carlo code described in [6]. A good agreement between the analytic formula and the simulation results is evident in Fig. 2.

Finally, we consider a general electric-field profile given by $E(z, \omega t)$, where $E(z, T)$ is $2 \pi$ periodic in $T$.

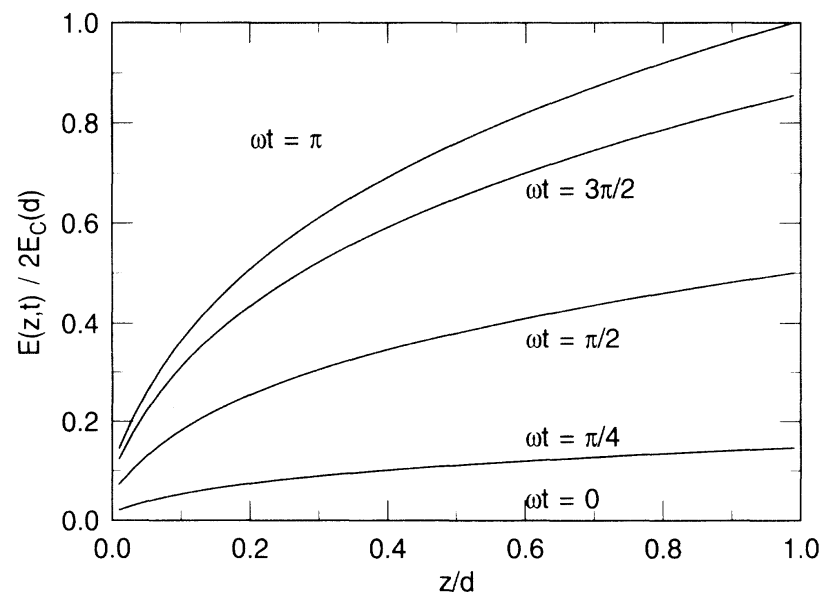

FIG. 1. The electric-field profile $E(z, \omega t)=E_{C}(z)(1+\cos \omega t)$ for different phases $\omega t$. Here $E_{C}(z)$ is given by Eq. (17) with $\alpha=20.3$ and $\beta=12.8$.
Defining the dc-bias field $E_{0}(z)=\overline{E(z, \tau)}$ and the rf component $E_{1}(z, \omega t)=E(z, \omega t)-E_{0}(z)$, we have the equation of motion for an ion in the sheath

$$
\frac{d^{2} \zeta}{d \tau^{2}}=\epsilon^{2}\left[F_{0}(\zeta)+F_{1}(\zeta, \tau+\phi)\right]
$$

where $\phi=\omega t_{l}, F_{0}=E_{0} / E_{0}(d)$, and $F_{1}=E_{1} / E_{0}(d)$, as before, and $F_{1}(\zeta, T)$ is $2 \pi$ periodic in $T$.

Before proceeding to the two-time-scale analysis, we introduce the following time-integral operator $\mathcal{L}$ :

$$
\mathcal{L} f=\int_{0}^{\tau} f\left(\tau^{\prime}\right) d \tau^{\prime}-\overline{\int_{0}^{\tau} f\left(\tau^{\prime}\right) d \tau^{\prime}} .
$$

If the function $f(\tau)$ is $2 \pi$ periodic in $\tau$ and satisfies $\bar{f}=0$, then the function $\mathcal{L} f$ is also $2 \pi$ periodic in $\tau$ and satisfies $\overline{\mathcal{L} f}=0$.

As in the case of Eq. (1), we consider the solutions of Eq. (18) for small $\epsilon$. In the limit $\epsilon \rightarrow 0$, we obtain Eq. (2) for $\zeta_{0}(\epsilon \tau)$. Writing $\zeta=\zeta_{0}(\epsilon \tau)+\epsilon^{2} \xi$, and employing the two-time-scale expansion scheme given in Eq. (4), we obtain the lowest-order solution $\xi_{0}=\mathcal{L}^{2} E_{1}(\tau+\phi)$ $+D_{0}(\tilde{\tau})$. Here $D_{0}(\tilde{\tau})$ denotes an integration constant (with respect to $\tau$ ), to be determined by a higher-order equation.

We may derive the ponderomotive force by taking the

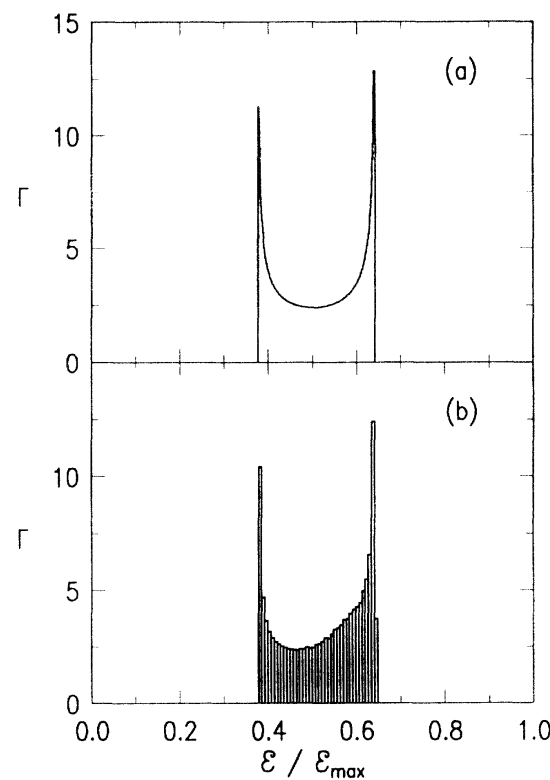

FIG. 2. The energy distributions of the ion flux at the cathode obtained from (a) the analytical expression Eq. (15), and (b) a numerical simulation based on Eq. (1). The electric field $E(z, \omega t)$ is given in Fig. 1, and the parameters used here are $u_{I}=v_{l} / d \omega=0.02$ and $\epsilon=0.16$, which correspond to the case where $\omega / 2 \pi=13.0 \mathrm{MHz}, d=0.83 \mathrm{~mm}$, and the dc-bias sheath potential $-\psi_{C}(d)=35 \mathrm{~V}$. In (a) the singularities are truncated by plotting $\bar{\Gamma}(\mathscr{E})$ for $\mathscr{E}-+\delta \mathscr{E} \leq \mathscr{E} \leq \mathscr{E}_{+}-\delta \mathscr{E}$ with $\delta \mathscr{E} /$ $\mathscr{E}_{\text {max }}=0.007$. In each case, $\bar{\Gamma}$ is normalized so as to enclose unit area. 
time average of the ion trajectory, i.e., taking steps similar to Eqs. (6) and (7). In the case of a general rf electric field, the equation for the time-average trajectory $\bar{\zeta}$ - corresponding to Eq. (8) - is given by

$$
\frac{d^{2} \bar{\zeta}}{d \tilde{\tau}^{2}}=F_{0}(\bar{\zeta})-\frac{\epsilon^{2}}{2} \frac{d \overline{\left(\mathcal{L E} E_{1}\right)^{2}}}{d \zeta}(\bar{\zeta})+\mathcal{O}\left(\epsilon^{3}\right) \text {. }
$$

As before, the last term of Eq. (19) represents the ponderomotive force.

The energy distribution may be obtained in a manner similar to that of deriving Eq. (15). We obtain the final velocity $v_{f}$ up to order $\epsilon^{2}$ as

$$
v_{f}=v^{\prime} 0 f+\left.(q / m \omega) \mathcal{L} E_{1}\right|_{z=d} .
$$

From Eq. (14) for the energy distribution of the ion flux, we obtain

$$
\begin{aligned}
\bar{\Gamma}(\mathscr{E}) & =\frac{n_{i} v_{I}}{\pi v^{\prime} 0 f}\left(\frac{m}{2}\right)^{1 / 2} \int \frac{\left(\mathscr{E}_{\varphi}\right)^{1 / 2} \delta(g) d g}{d \mathscr{E}_{\varphi} / d \varphi} \\
& =\frac{n_{i} v_{l}}{\pi v^{\prime} \rho f}\left(\frac{m \mathscr{E}}{2}\right)^{1 / 2} \sum_{i}\left|\frac{d \mathscr{E}_{\varphi}}{d \varphi}\right|_{\varphi_{i}}^{-1},
\end{aligned}
$$

where $\mathscr{E}_{\varphi}=m v_{f}^{2} / 2$ denotes the final kinetic energy as a function of the final phase $\varphi=\omega t, g=(2 \mathscr{E} / m)^{1 / 2}-\left(2 \mathscr{E}_{\varphi} /\right.$ $m)^{1 / 2}$, and the discrete phases $\varphi_{i}=\varphi_{i}(\mathscr{E})$ denote all the distinct solutions $\varphi$ of the equation $\mathscr{E}=\mathscr{E}_{\varphi}(\varphi)$. In the case of sinusoidal time dependence, the general expression reduces to a simple closed-form formula for $\bar{\Gamma}(\mathscr{E})$, as given by Eq. (15). In general, however, the energy distribution $\bar{\Gamma}(\mathscr{E})$ has an "implicit" form, defined by Eq. (21). Note that, corresponding to any value of the energy $\mathscr{E}$ where $d \mathscr{E}_{\varphi} / d \varphi$ vanishes, there will be a singularity in the ion energy distribution $\bar{\Gamma}(\mathscr{E})$.

In conclusion, we have derived an analytical expression, appropriate in the high-frequency regime, for the energy distribution $\bar{\Gamma}(\mathscr{E})$ of the ion flux in a collisionless rf sheath. We have also noted that the ponderomotive force due to the oscillating sheath field exerts a retarding influence on the ion trajectories. In real rf sheaths, an oscillatory motion of the relatively sharp presheath-sheath boundary can be expected. Although in principle the discussion of a general electric field $E(z, \omega t)$ yields an ener- gy distribution, defined by Eq. (21), for a sheath with such an oscillating sharp boundary, a more detailed analysis of such a model will be treated in a separate paper [20].

[1] B. Chapman, Glow Discharge Processes (Wiley, New York, 1980).

[2] S. M. Sze, VSLI Technology (McGraw-Hill, New York, 1988).

[3] D. B. Graves, J. Appl. Phys. 62, 88 (1987).

[4] M. A. Lieberman, IEEE Trans. Plasma Sci. 6, 638 (1988).

[5] T. J. Sommerer, W. N. Hitchon, and J. E. Lawler, Phys. Rev. Lett. 63, 2361 (1989).

[6] R. T. Farouki, S. Hamaguchi, and M. Dalvie, Phys. Rev. A 44, 2664 (1991).

[7] S. Hamaguchi, R. T. Farouki, and M. Dalvie, Phys. Rev. A 44, 3804 (1991).

[8] J. W. Coburn and E. Kay, J. Appl. Phys. 43, 4965 (1972).

[9] W. M. Holber and J. Forster, J. Vac. Sci. Technol. A 8, 3720 (1990).

[10] M. J. Kushner, J. Appl. Phys. 58, 4024 (1985).

[11] B. E. Thompson, H. H. Sawin, and D. A. Fisher, J. Appl. Phys. 63, 2241 (1988).

[12] A. Metze, D. W. Ernie, and H. J. Oskam, J. Appl. Phys. 65, 4024 (1989).

[13] D. Vender and R. Boswell, IEEE Trans. Plasma Sci. 18, 725 (1990).

[14] M. S. Barnes, J. C. Forster, and J. H. Keller, IEEE Trans. Plasma Sci. 19, 240 (1991).

[15] P. Benoit-Cattin and L. C. Bernard, J. Appl. Phys. 39, 5723 (1968).

[16] C. D. Child, Phys. Rev. 32, 492 (1911); I. Langmuir, Phys. Rev. 2, 450 (1913).

[17] J. Kevorkian and J. D. Cole, Perturbation Methods in Applied Mathematics (Springer-Verlag, New York, 1968).

[18] F. Chen, Introduction to Plasma Physics (Plenum, New York, 1974).

[19] R. T. Farouki, M. Dalvie, and L. F. Pavarino, J. Appl. Phys. 68, 6106 (1990).

[20] R. T. Farouki, S. Hamaguchi, and M. Dalvie (to be published). 